See discussions, stats, and author profiles for this publication at: https://www.researchgate.net/publication/305252610

\title{
Soret and Dufour effects on MHD viscoelastic fluid flow through a vertical flat plate with constant suction
}

Conference Paper · July 2016

DOI: $10.1063 / 1.4958367$

CITATIONS

0

2 authors:

(26) Sheikh Imamul Hossain

1. University of Technology Sydney

9 PUBLICATIONS 19 CITATIONS

SEE PROFILE
READS

45

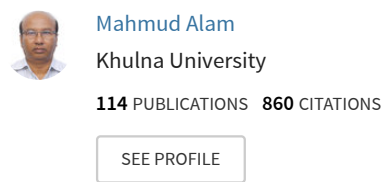

Some of the authors of this publication are also working on these related projects:

Computational Modelling and Simulation of Fluid-structures and Multi-phase Flows View project

Unsteady MHD Bingham Fluid Flow through a Parallel Plate with Ion slip and Hall Current View project 


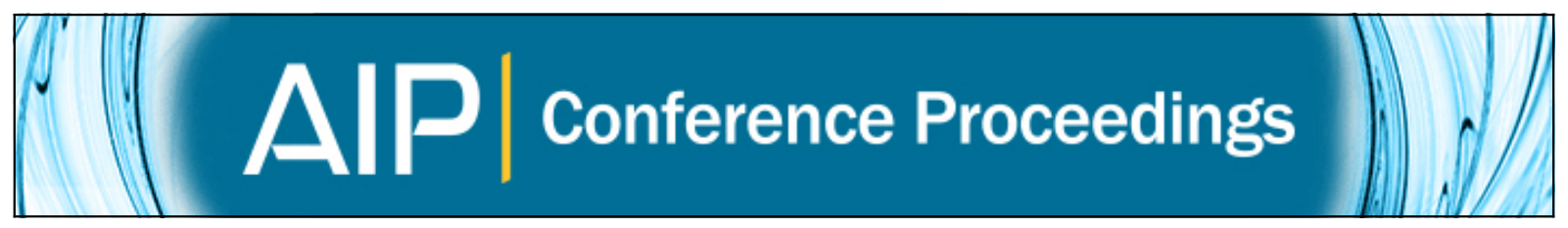

Soret and Dufour effects on MHD viscoelastic fluid flow through a vertical flat plate with constant suction

Sheikh Imamul Hossain and Md. Mahmud Alam

Citation: AIP Conference Proceedings 1754, 040007 (2016); doi: 10.1063/1.4958367

View online: http://dx.doi.org/10.1063/1.4958367

View Table of Contents: http://scitation.aip.org/content/aip/proceeding/aipcp/1754?ver=pdfcov

Published by the AIP Publishing

\section{Articles you may be interested in}

MHD Couette flow of viscous incompressible fluid with Hall current and suction

AIP Conf. Proc. 1754, 040006 (2016); 10.1063/1.4958366

On the double diffusive convection flow of Eyring-Powell fluid due to cone through a porous medium with Soret and Dufour effects

AlP Advances 5, 057140 (2015); 10.1063/1.4921488

Dufour effect on unsteady natural convection flow past an infinite vertical plate with constant heat and mass fluxes

AIP Conf. Proc. 1621, 470 (2014); 10.1063/1.4898508

On thermosolutal convection in micropolar fluid in porous medium: Soret-Dufour phenomenon

AIP Conf. Proc. 1453, 197 (2012); 10.1063/1.4711175

Dufour and Soret effects in a magnetized and nonmagnetized plasma

Phys. Plasmas 14, 012305 (2007); 10.1063/1.2428279 


\title{
Soret and Dufour Effects on MHD Viscoelastic Fluid Flow Through a Vertical Flat Plate with Constant Suction
}

\author{
Sheikh Imamul Hossain ${ }^{\mathrm{a}}$ and Md. Mahmud Alam ${ }^{\mathrm{b}}$ \\ Mathematics Discipline, Khulna University, Khulna-9208 \\ ${ }^{\mathrm{b}}$ Corresponding author: alam_mahmud2000@yahoo.com \\ as.imamul.ku@gmail.com
}

\begin{abstract}
An attempt is made to represent the numerical solution of magnetohydrodynamics (MHD) viscoelastic fluid flow through an infinite vertical flat plate with constant suction in the presence of Soret and Dufour effects. The expressions of nondimensional, coupled partial momentum, energy and concentration differential equations are obtained with the help of the usual non-dimensional variables. Implicit finite difference method is imposed to obtain the non-dimensional equations. Also the stability conditions and convergence criteria are analyzed. The effects of the various parameters entering into the problem on shear stress, Nusselt number, and Sherwood number are demonstrated graphically with physical interpretation.
\end{abstract}

\section{INTRODUCTION}

The most common non-Newtonian fluid is viscoelastic fluid. It has two properties, one is viscous property and another one is elastic property at the time of undergoing deformation and so it is named as viscoelastic fluids. Its importance is increasing day by day due to its many engineering, biological, industrial and chemical aspects. Some common viscoelastic fluids are engine oils, paints, honey, shampoo, ointments, gels, molten plastics, blood and so on. These appear in many industrial process, chemical reaction and pharmaceutical industries.

From the above point of applications, the analysis of viscoelastic fluids started about 1964 by Beard and Walters [1]. Rajagopal et al. [2] studied the boundary layer flow of a viscoelastic fluid over a stretching sheet. The study of boundary layer flow of a viscoelastic fluid through a vertical plate has wide range of applications in the field of chemical engineering and production of synthetic sheets. This is also consequential to the production of heavy crude oils by means of thermal process. Heat and mass transfer analysis are the fundamental scopes in fluid dynamics analysis. Flow and heat transfer of a viscoelastic fluid over a flat plate with a magnetic field and a pressure gradient has been analyzed by kumar et al. [3]. Dufour and Soret Effects on unsteady MHD Free Convection and mass transfer fluid flow through a porous medium in a rotating system have been investigated by Islam and Alam [4]. Heat and mass transfer for Soret and Dufour's effect on mixed convection boundary layer flow over a stretching vertical surface in a porous medium filled with a viscoelastic fluid in the presence of magnetic field has been examined by Gbadeyan et al. [5]. Hossain and Alam [6] have extended the work of Gbadeyan et al. [5] by implicit finite difference method.

Hence our aim of this work is to extend the work of Hossain and Alam [6] when a magnetic field is imposed in a direction which is perpendicular to the free stream. The problem has been solved by implicit finite difference method. The governing equations involved in this problem have been transformed into non-similar coupled partial differential equations by usual transformations. Stability and convergence criteria are analyzed for finding the restriction of the parameters. Finally, the comparison of the present results with the results of Gbadeyan et al. [5] has been discussed. 


\section{MATHEMATICAL MODEL}

Consider the unsteady one-dimensional laminar flow of an incompressible viscoelastic fluid (obeying second grade model) through a vertical plate $y=0$ with Soret and Dufour effects. The positive $x$ coordinate is measured along the plate in the direction of fluid motion and the positive $y$ coordinate is measured normal to the plate. Since the plate is infinite and the motion is unsteady so all the flow variables depend only upon $y$ and time $t$. A uniform magnetic field $\mathbf{B}$ is imposed to the plate $(y=0)$ to be acting along the $y$-axis which is assumed to be electrically non-conducting. Assumed that $\mathbf{B}=\left(B_{x}, B_{y}, B_{z}\right)=\left(0, B_{0}, 0\right)$ is the magnetic field vector. The magnetic Reynolds number of the flow is taken to be small so that the induced magnetic field is negligible in comparison with the applied magnetic field. The variable temperature $T_{w}$ and variable concentration $C_{w}$ at wall of the plate occupied with viscoelastic fluid of uniform ambient temperature $T_{\infty}$ and uniform ambient concentration $C_{\infty}$. The physical configuration of the above problem is shown in FIGURE 1.

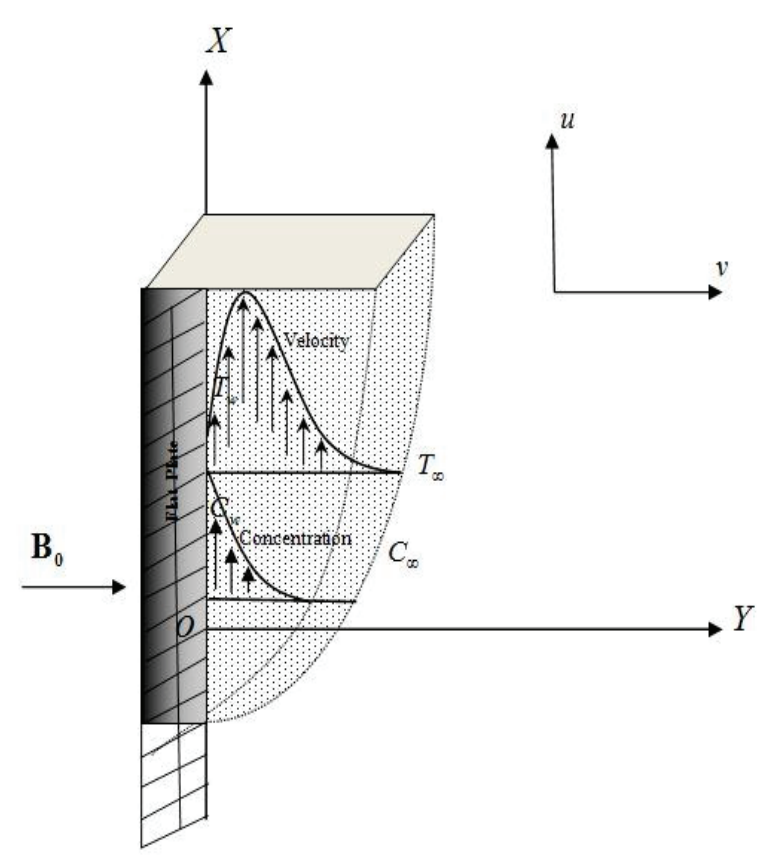

FIGURE 1. Physical configuration of the flow

Within the framework of the above state assumptions and using the dimensionless quantities $\bar{U}=\frac{u}{U_{0}}$, $Y=\frac{y U_{0}}{v}, \tau=\frac{t U_{0}^{2}}{v}, \bar{\theta}=\frac{T-T_{\infty}}{T_{w}-T_{\infty}}$ and $\bar{\phi}=\frac{C-C_{\infty}}{C_{w}-C_{\infty}}$ in the equations relevant to the problem is governed by the following coupled non-linear non-dimensional partial differential equations under the electromagnetic Boussinesq approximations as;

$$
\begin{aligned}
& \frac{\partial \bar{U}}{\partial \tau}-S \frac{\partial \bar{U}}{\partial Y}=G_{r} \bar{\theta}+G_{m} \bar{\phi}+\frac{\partial^{2} \bar{U}}{\partial Y^{2}}-M \bar{U}+K\left[\frac{\partial^{3} \bar{U}}{\partial \tau \partial Y^{2}}-S \frac{\partial^{3} \bar{U}}{\partial Y^{3}}\right] \\
& \frac{\partial \bar{\theta}}{\partial \tau}-S \frac{\partial \bar{\theta}}{\partial Y}=\frac{1}{P_{r}} \frac{\partial^{2} \bar{\theta}}{\partial Y^{2}}+M E_{c} \bar{U}^{2}+D_{u} \frac{\partial^{2} \bar{\phi}}{\partial Y^{2}} \\
& \frac{\partial \bar{\phi}}{\partial \tau}-S \frac{\partial \bar{\phi}}{\partial Y}=\frac{1}{S_{c}} \frac{\partial^{2} \bar{\phi}}{\partial Y^{2}}+S_{r} \frac{\partial^{2} \bar{\theta}}{\partial Y^{2}}
\end{aligned}
$$

The corresponding boundary conditions are;

$$
\begin{aligned}
& \bar{U}=1, \bar{\theta}=1, \bar{\phi}=1 \text { at } Y=0 \\
& \bar{U} \rightarrow 0, \bar{\theta} \rightarrow 0, \bar{\phi} \rightarrow 0 \text { as } Y \rightarrow \infty
\end{aligned}
$$

where, Grashof number of heat transfer, $G_{r}=\frac{v g \beta\left(T_{w}-T_{\infty}\right)}{U_{0}^{3}}$; Grashof number of mass transfer, $G_{m}=\frac{v g^{*} \beta\left(C_{w}-C_{\infty}\right)}{U_{0}^{3}} ;$ Dimensionless Viscoelastic Parameter, $K=k_{0} \frac{U_{0}^{2}}{v^{2}} ;$ Magnetic Parameter, $M=\frac{1}{\rho} \frac{\sigma B_{0}{ }^{2} v}{U_{0}{ }^{2}} ;$ 
Suction parameter, $S=\frac{v_{0}}{U_{0}}$; Prandtl number, $P_{r}=\frac{v \rho c_{p}}{k}$; Eckert Number, $E_{c}=\frac{U_{0}^{2}}{c_{p}\left(T_{w}-T_{\infty}\right)}$; Dufour number, $D_{u}=\frac{D_{m}}{v} \frac{\left(C_{w}-C_{\infty}\right)}{\left(T_{w}-T_{\infty}\right)} ;$ Schmidt number, $S_{c}=\frac{v}{D_{m}} ;$ Soret number, $S_{r}=\frac{D_{T}}{v} \frac{\left(T_{w}-T_{\infty}\right)}{\left(C_{w}-C_{\infty}\right)}$.

\section{SHEAR STRESS, NUSSELT NUMBER AND SHERWOOD NUMBER}

From the velocity, the effects of various parameters on the local and average shear stress have been calculated. The following equation represents the local shear stress at the plate. Local shear stress $\tau_{L}=\mu\left(\frac{\partial u}{\partial y}\right)_{y=0}$ which is proportional to $\left(\frac{\partial \bar{U}}{\partial Y}\right)_{Y=0}$. From the temperature field, the effects of various parameters on the local heat transfer coefficients have been investigated. The following equation represents the local heat transfer rate that is well known Nusselt number. Local Nusselt number, $N_{u L}=\mu\left(-\frac{\partial T}{\partial y}\right)_{y=0}$ which is proportional to $\left(-\frac{\partial \bar{\theta}}{\partial Y}\right)_{Y=0}$. From the concentration field, the effects of various parameters on the local mass transfer coefficients have been analyzed. The following equation represents the local mass transfer rate that is well known Sherwood number. Local Sherwood number, $S_{h L}=\mu\left(-\frac{\partial C}{\partial y}\right)_{y=0}$ which is proportional to $\left(-\frac{\partial \bar{\phi}}{\partial Y}\right)_{Y=0}$.

\section{NUMERICAL ANALYSIS}

To solve the non-dimensional system by implicit finite difference technique, it is required to set finite difference equations. Here, the region within the boundary layer is divided by some perpendicular lines of $Y-$ axis, where $Y$-axis is normal to the medium as shown in FIGURE 2. It is assumed that the maximum length of boundary layer is $Y_{\max }(=35)$ as corresponding to $Y \rightarrow \infty$ i. e., $Y$ varies from 0 to 35 and the number of grid spacing in $Y$ direction is 200 . The constant mesh along $Y$ axis becomes $\Delta Y=0.175(0 \leq Y \leq 35)$ with the smaller time step $\Delta \tau=0.001$.

Let $\bar{U}_{i}^{n+1}, \bar{\theta}_{i}^{n+1}$ and $\bar{\phi}_{i}^{n+1}$ denote the and values of $\bar{U}_{i}^{n}, \bar{\theta}_{i}^{n} \bar{\phi}_{i}^{n}$ at the end of a time-step respectively. Using the implicit finite difference approximation and the system of partial differential equations (5)-(8) an appropriate set of finite difference equations have been obtained as;

$$
\begin{aligned}
& \frac{\bar{U}_{i}^{n+1}-\bar{U}_{i}^{n}}{\Delta \tau}-S \frac{\bar{U}_{i+1}^{n}-\bar{U}_{i}^{n}}{\Delta Y}=G_{r} \bar{\theta}_{i}^{n}+G_{m} \bar{\phi}_{i}^{n}+\frac{\bar{U}_{i+1}^{n}-2 \bar{U}_{i}^{n}+\bar{U}_{i-1}^{n}}{(\Delta Y)^{2}}-M \bar{U}_{i}^{n} \\
& +K\left[\frac{\bar{U}_{i+1}^{n+1}-2 \bar{U}_{i}^{n+1}+\bar{U}_{i-1}^{n+1}-\bar{U}_{i+1}^{n}+2 \bar{U}_{i}^{n}-\bar{U}_{i-1}^{n}}{\Delta \tau(\Delta Y)^{2}}-S \frac{\bar{U}_{i+3}^{n}-3 \bar{U}_{i+2}^{n}+3 \bar{U}_{i+1}^{n}-\bar{U}_{i}^{n}}{(\Delta Y)^{3}}\right] \\
& \frac{\bar{\theta}_{i}^{n+1}-\bar{\theta}_{i}^{n}}{\Delta \tau}-S \frac{\bar{\theta}_{i+1}^{n}-\bar{\theta}_{i}^{n}}{\Delta Y}=\frac{1}{P_{r}} \frac{\bar{\theta}_{i+1}^{n}-2 \bar{\theta}_{i}^{n}+\bar{\theta}_{i-1}^{n}}{(\Delta Y)^{2}}+M E_{c} \bar{U}_{i}^{2 n}+D_{u} \frac{\bar{\phi}_{i+1}^{n}-2 \bar{\phi}_{i}^{n}+\bar{\phi}_{i-1}^{n}}{(\Delta Y)^{2}}
\end{aligned}
$$


$\frac{\bar{\phi}_{i}^{n+1}-\bar{\phi}_{i}^{n}}{\Delta \tau}-\frac{\bar{\phi}_{i+1}^{n}-\bar{\phi}_{i}^{n}}{\Delta Y}=\frac{1}{S_{c}} \frac{\bar{\phi}_{i+1}^{n}-2 \bar{\phi}_{i}^{n}+\bar{\phi}_{i-1}^{n}}{(\Delta Y)^{2}}+S_{r} \frac{\bar{\theta}_{i+1}^{n}-2 \bar{\theta}_{i}^{n}+\bar{\theta}_{i-1}^{n}}{(\Delta Y)^{2}}$

The boundary conditions with the finite difference scheme are as;

$\bar{U}_{i}^{n}=1, \bar{\theta}_{i}^{n}=1, \bar{\phi}_{i}^{n}=1$

$\bar{U}_{L}^{n}=0, \bar{\theta}_{L}^{n}=0, \bar{\phi}_{L}^{n}=0$ where $L \rightarrow \infty$

Here the subscript $i$ degenerate the grid points with $Y$ coordinates and superscript $n$ represents the value of time, $\tau=n \Delta \tau$, where, $n=0,1,2, \ldots \ldots$. The velocity $(\bar{U})$, temperature $(\bar{\theta})$ and concentration $(\bar{\phi})$ distributions at all interior nodal points may be computed by successive applications of the above finite difference equations. The numerical values of the local Shear Stress, Nusselt number and Sherwood number are evaluated by five-point approximate formula for the derivatives. The stability condition and convergence criteria of the problem are as furnished as $P_{r} \geq 0.07$ and $S_{c} \geq 0.07$ which are not shown in details for brevity.

\section{RESULTS AND DISCUSSION}

In order to reveal the steady-state solutions, the computations have been carried out up to dimensionless time $\tau=120$. It is observed that the numerical values of $\bar{U}, \bar{\theta}$ and $\bar{\phi}$ however, show a little changes after dimensionless time $\tau=60$. Thus the solutions for dimensionless time $\tau=60$ are essentially steady-state solutions. It has been seen that the graphical representation of various dimensionless parameters have been reached its highest convergence when mesh size is taken as $n=200$ (FIGURE 3(a) and 3(b)). Therefore the flow parameters have been illustrated graphically in FIGURES (4-5) with dimensionless time $\tau=60$ and mesh size $n=200$. For brevity, effects of two parameters have been illustrated.

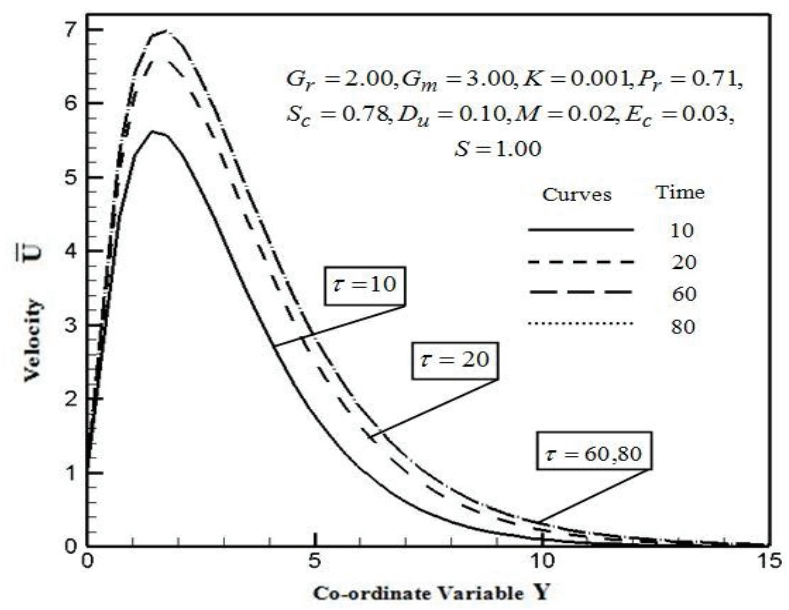

(a)

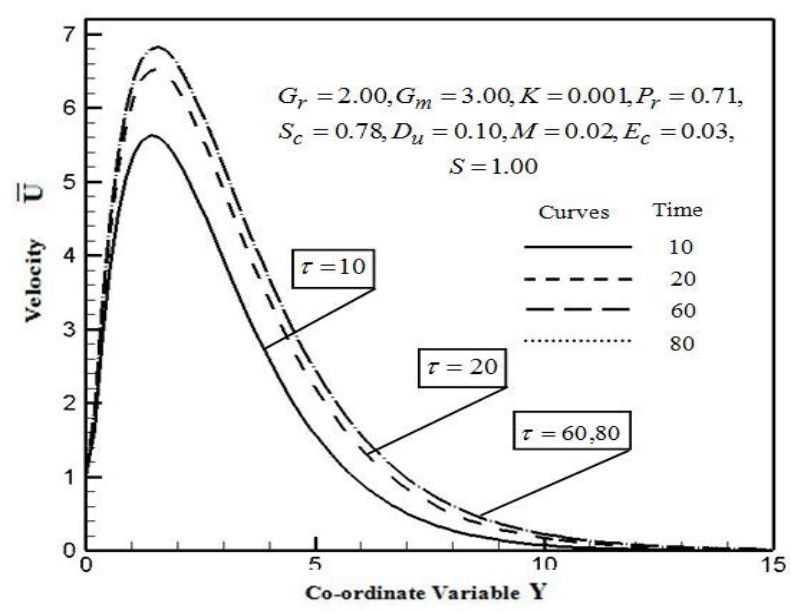

(b)

FIGURE 3. Steady state solution for $S_{r}=2.00$ at different time intervals with (a) mesh size $n=100$ (b) mesh size $n=200$

The graphical results are plotted from numerical solutions by using the computational software Tecplot 9.0. FIGURES 4(a-b) are plotted to present the influence of Dufour number $D_{u}$ on both fluid velocity and shear stress.

It is noted that in FIGURE 4(a) the velocity of the fluid has been increased as Dufour number $D_{u}$ increased. A similar behavior is also expected in FIGURE 4(b) due to the fact that increasing the velocity means increasing the shear stress for a certain parameter. In FIGURE 4(b), the shear stress increases with the increase of Dufour number $D_{u}$. 


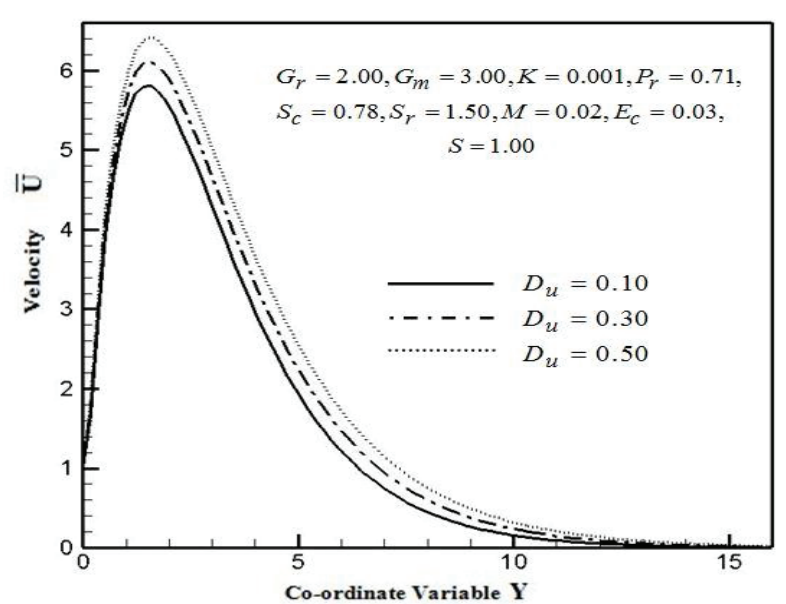

(a)

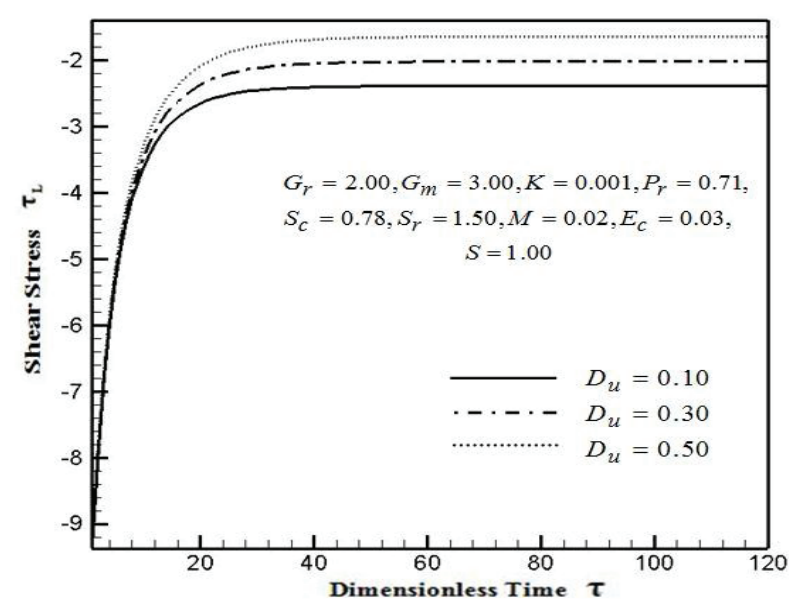

(b)

FIGURE 4. (a) Velocity profiles (b) Shear stress for different values of Dufour number $D_{u}$

FIGURES 5(a-b) are plotted to show the effect of Soret number $S_{r}$ on fluid velocity and shear stress. FIGURE 5 (a) depicts that the fluid velocity has been seen increasing effect due to increase of Soret number $S_{r}$. FIGURE 5 (b) is illustrated to display the effect of Soret number $S_{r}$ on fluid shear stress. It is clearly seen that, fluid shear stress has been increased with the increase of Soret number $S_{r}$.

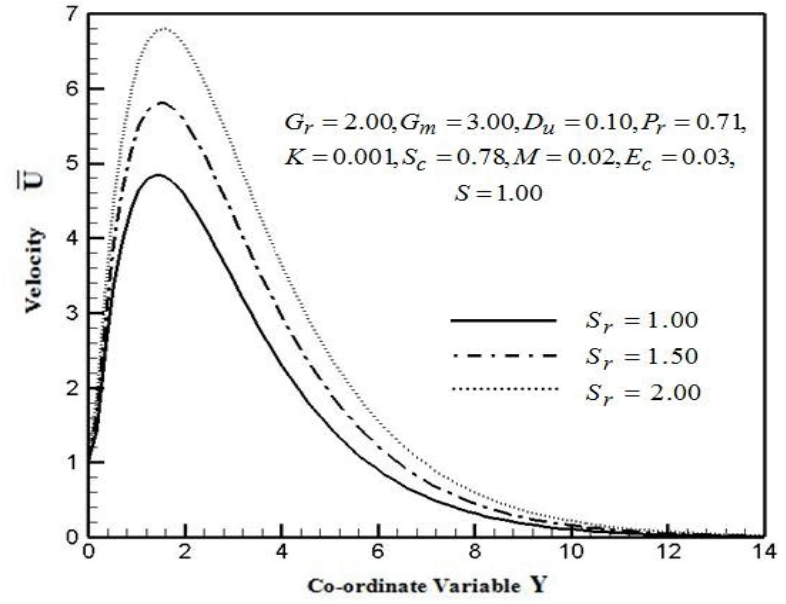

(a)

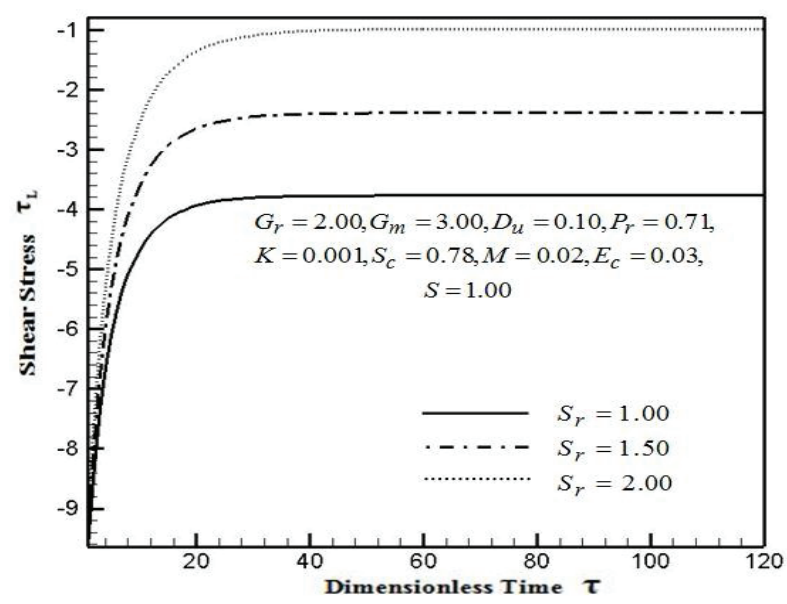

(b)

FIGURE 5. (a) Velocity profiles (b) Shear stress for different values of Soret number $S_{r}$

\section{COMPARISON}

Qualitative comparison of the present results with previous results is presented in a tabular form.

TABLE 1. Qualitative comparison of the present results with the previous results

\begin{tabular}{ccc}
\hline Increased Parameter & $\begin{array}{c}\text { Previous Results Given By } \\
\text { Gbadeyan et al.[5] }\end{array}$ & Present Results \\
\hline$D_{u}$ & Increase & Increase \\
$S_{r}$ & Increase & Increase \\
\hline
\end{tabular}


Finally, a qualitative comparison of the present steady-state results with the published results (Gbadeyan et al. [5]) is presented graphically in FIGURES 6(a-b).

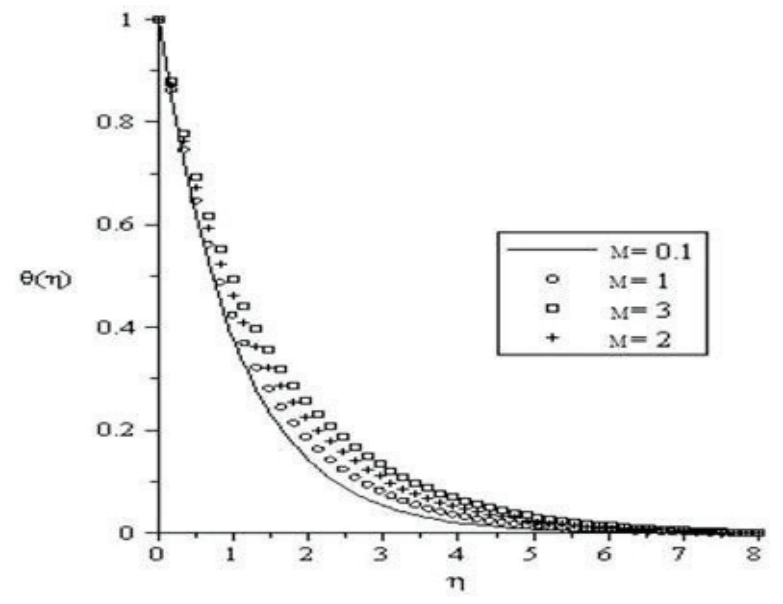

(a)

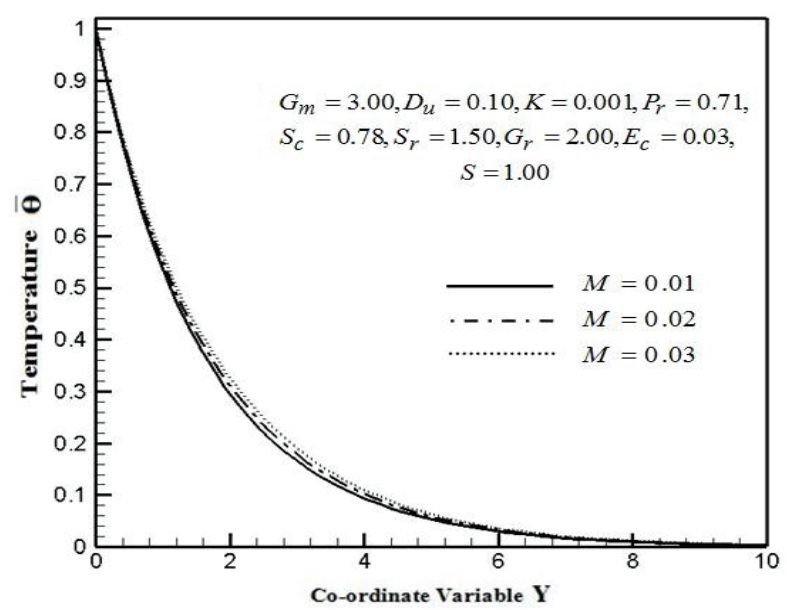

(b)

FIGURE 6. Temperature profiles (a) Published result (Gbadeyan et al. [5]) (b) Present result for different values of Magnetic parameter $M$

The results are seen qualitatively same behavior but quantitatively different. In this work, the implicit finite difference method has been used as a solution technique but Gbadeyan et al. [5] has used similarity technique with shooting method as a solution technique.

\section{CONCLUSIONS}

Implicit finite difference method has been applied to solve the model and also shown the effects of Soret and Dufour number on MHD viscoelastic fluid flow through a vertical flat with constant suction. The accuracy of the obtained results is checked through the imposed conditions and graphs. The obtained results are qualitatively good in case of all the flow parameters but not shown for brevity. The following main results are concluded from this study;

1. For the increase of Dufour number $D_{u}$, the velocity and the shear stress have been increased.

2. Velocity and shear stress of the fluid have been increased with the increase of Soret number $S_{r}$.

\section{ACKNOWLEDGMENTS}

This work is financed and supported by National Science and Technology (NST) under Ministry of Science and Technology, Government of the People's Republic Bangladesh.

\section{REFERENCES}

1. D.W. Beard and K. Walters, Mathematical Proceedings of the Cambridge Philosophical Society 60(3), 667-674 (1964).

2. K. R. Rajagopal, T. Y. Na, A. S. Gupta, Rheologica Acta 23, 213-215 (1984).

3. M. Kumar, H.S. Takhar, G. Nath, Indian Journal of pure and applied Mathematics 28(1), 109-121 (1997).

4. N. Islam and M.M. Alam, Bangladesh Journal of Science and Industrial Research 43(2), 159-172 (2008).

5. J.A. Gbadeyan, A.S. Idowu, A.W. Ogunsola, O.O. Agboola, P.O. Olanrewaju, Global Journal of science Frontier Research 11(8), 96-114 (2011).

6. S.I. Hossain and M.M. Alam, Procedia Engineering 105, 309-316 (2015). 